\title{
Inframalleolar Bypass to the Distal Portion of the Lateral Tarsal Artery for Limb Salvage
}

\author{
Sosei Kuma, MD, PhD, 1,2 Koichi Morisaki, MD, PhD, 2,3 Jin Okazaki, MD, PhD, ${ }^{2}$ \\ and Shinsuke Mii, MD, PhD2,4
}

\begin{abstract}
A 72-year-old man was admitted to our hospital due to rest pain and gangrene on his left second foot digit. Angiography revealed continuous patency from the superficial femoral artery stent to the below-knee popliteal artery with a diffuse, occlusive lesion in the crural arteries. The distal portion of the lateral tarsal artery was patent. Popliteal to lateral tarsal artery bypass was performed, and an immediate amputation of the second foot digit resulted in secondary healing. Vascular surgeons should consider the distal portion of the lateral tarsal artery as an effective alternative target for infragenicular revascularization.
\end{abstract}

Keywords: distal bypass, paramalleolar artery, foot salvage

\section{Introduction}

Surgical revascularization of the paramalleolar artery is a powerful option for limb salvage. Although paramalleolar artery bypasses are usually performed, ${ }^{1,2)}$ some cases in which both the dorsal pedal artery (DPA) and the paramalleolar posterior tibial artery (PTA) are occluded require bypass to the branches of these vessels (i.e., the

\footnotetext{
${ }^{1}$ Department of Vascular Surgery, Fukuoka-Higashi Medical Center, Koga, Fukuoka, Japan

${ }^{2}$ Department of Vascular Surgery, Kokura Memorial Hospital, Kitakyushu, Fukuoka, Japan

${ }^{3}$ Department of Surgery and Science, Graduate School of Medical Sciences, Kyushu University, Fukuoka, Japan

${ }^{4}$ Department of Vascular Surgery, Saiseikai Yahata General Hospital, Kitakyushu, Fukuoka, Japan
}

Received: September 26, 2018; Accepted: November 7, 2018 Corresponding author: Sosei Kuma, MD, PhD. Department of Vascular Surgery, Fukuoka-Higashi Medical Center, 1-1-1 Chidori, Koga, Fukuoka 811-3195, Japan

Tel: +81-92-943-2331, Fax: +81-92-943-8775

E-mail: kumasosei@yahoo.co.jp

(cc) BY-NC-SA (02019 The Editorial Committee of Annals of Vascular Diseases. This article is distributed under the terms of the Creative Commons Attribution License, which permits use, distribution, and reproduction in any medium, provided the credit of the original work, a link to the license, and indication of any change are properly given, and the original work is not used for commercial purposes. Remixed or transformed contributions must be distributed under the same license as the original. lateral tarsal and plantar arteries).

We herein report a case of critical limb ischemia (CLI) that was treated with inframalleolar bypass to the distal portion of the lateral tarsal artery (LTA). The patient provided their written consent for the publication of this case report.

\section{Case Report}

A 72-year-old man was admitted to our hospital due to rest pain and gangrene on his left second foot digit. He had a history of hypertension, diabetes mellitus (DM), end-stage renal disease, old cerebral infarction, and coronary artery disease. He had undergone coronary arterial bypass grafting, below-the-knee amputation of the right leg, and endovascular therapy (EVT) for coronary arteries, right carotid artery, right vertebral artery, right subclavian artery, and bilateral superficial femoral to crural arteries. A physical examination for left lower extremity performed on presentation was notable for dry gangrene of the second foot digit, popliteal arterial good pulsation, and diminished dorsal pedal and posterior tibial arterial pulsation. The skin perfusion pressure (SPP) was $17 \mathrm{mmHg}$ on the left plantar and $32 \mathrm{mmHg}$ on the left dorsal foot. Angiography revealed continuous patency from the superficial femoral artery stent to the below-knee popliteal artery (BKPA) and diffuse occlusive lesion in the crural arteries. On the ankle, the PTA was patent but too calcified to anastomose (Fig. 1). The distal portion of the LTA was patent (Fig. 2A).

Before the operation, the LTA was observed and marked with a duplex scan (Fig. 2B). Under general anesthesia, the BKPA was exposed and encircled, and intraoperative digital subtraction angiography (iDSA) was performed via the BKPA. As iDSA visualized the LTA in accordance with the preoperative marking, a skin incision was made along the marked portion. After confirming the location of the target artery using Doppler ultrasound, the extensor digitorum brevis muscle was cut (Fig. 3A), and then the LTA was exposed. Following systemic heparinization, arteriotomy was carried out on the BKPA, and a saphenous vein graft was anastomosed with 6-0 polypropylene continu- 
A

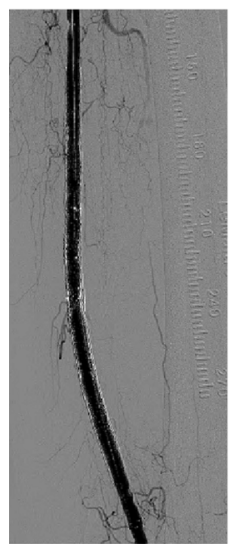

B

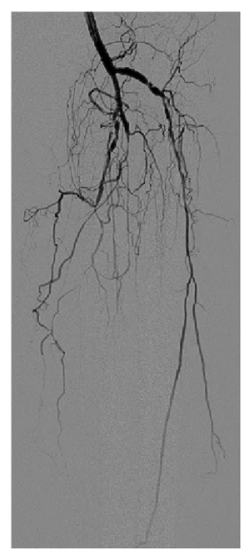

C

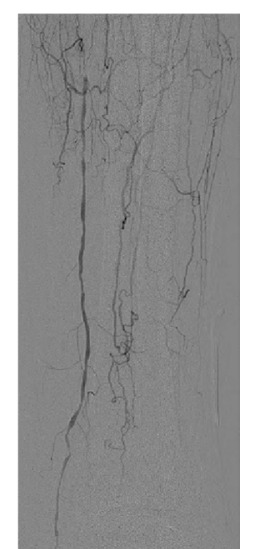

Fig. 1 Preoperative angiography.

(A) The superficial femoral artery stent was patent without restenosis. (B) The below-knee popliteal artery was patent, but tibial or peroneal arteries were occluded. (C) The distal portion of the posterior tibial artery was patent with multiple stenosis.

ous sutures. The distal end of the vein graft was conducted to the foot. Arteriotomy was carried out on the LTA, and the vein graft was anastomosed with 8-0 polypropylene continuous sutures. Amputation of the second foot digit was performed immediately after the bypass. Postoperative angiography showed that the popliteal-LTA bypass was patent and perfused the foot well (Fig. 3B). The SPP rose to $54 \mathrm{mmHg}$ on the left plantar and $51 \mathrm{mmHg}$ on the left dorsal foot, and the stump of the digit achieved secondary healing.

His postoperative course was uneventful, and he was discharged home 23 days after the operation. Although he died suddenly from an unknown cause 8 months after the operation, the primary patency of the bypass graft and limb salvage was successfully achieved for the remainder of his life.

\section{Discussion}

Distal vein bypass to the paramalleolar artery is now routine for most vascular surgeons. ${ }^{1,2)}$ This operation is most commonly performed to treat ischemic foot complications of DM, reflecting the unique pattern of atherosclerosis in these patients. ${ }^{3)}$

Occasionally, for patients in whom both the DPA and the paramalleolar PTA are occluded, the tarsal or plantar arteries may serve as runoff vessels if they remain patent. In the present case, the distal portion of the LTA was selected as the target vessel because the PTA was patent but highly calcified. However, in contrast to the PTA or DPA, there is little description concerning how to expose the LTA in textbooks, ${ }^{4}$ as it is an exceptional anastomotic site.

A

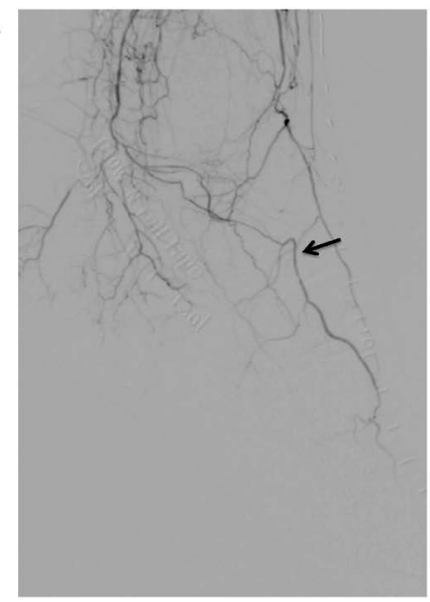

B

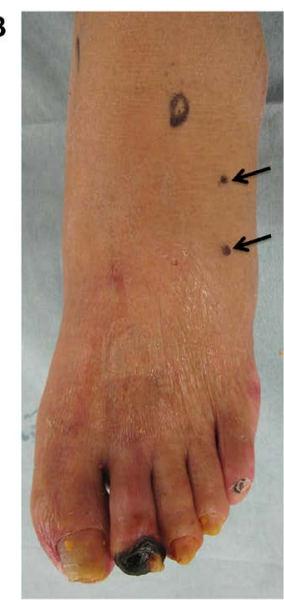

Fig. 2 Preoperative photography and angiography.

(A) The dry gangrene of left second digit was notable. The arrowheads indicate the lateral tarsal artery (LTA) marked using duplex scan. (B) The arrowheads indicate the LTA.
A

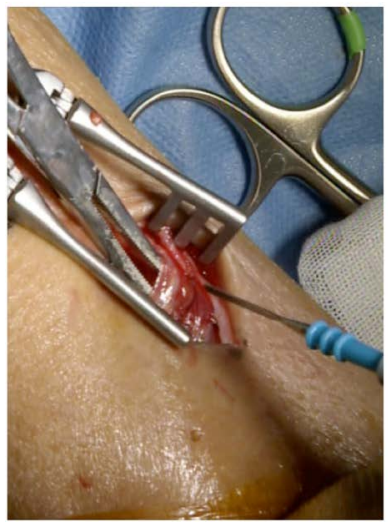

B

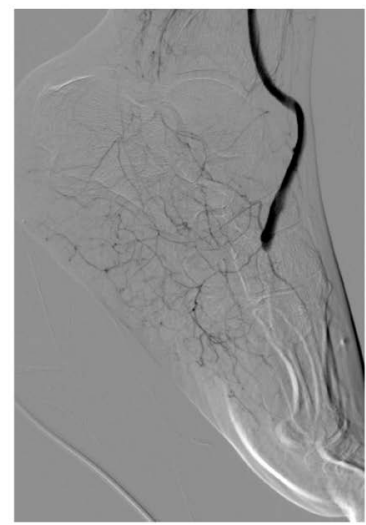

Fig. 3 Intraoperative photography and postoperative angiography.

(A) The extensor digitorum brevis muscle was cut. (B) The foot arteries were well perfused through the bypass to the lateral tarsal artery.

Hughes et al. described the surgical exposure of the LTA as follows $^{5)}$ : the DPA is exposed first; then, the takeoff point of the LTA is identified at the level of the navicular bone, and the incision is extended in a slightly lateral direction to expose it completely. In the present case, the patent portion of the LTA was quite far from the takeoff point. The present case required an extended incision when tracing from the takeoff of the LTA, an approach that might carry an increased risk of wound complications. Furthermore, if the position of the takeoff is accidentally missed, it might necessitate unnecessarily extensive dissection. Therefore, we directly exposed the distal portion of the LTA, assisted by preoperative US, iDSA, and intraoperative Doppler, and there were no wound complications. To our knowl- 
edge, the present case report might be the first detailed report concerning the distal bypass to the distal portion of the LTA reported in the English literature. This method might also be applicable for the exposure of other arteries unfamiliar to the surgeon.

Although EVT was primarily repeated for the present case because of his comorbidities, the revascularization became stuck due to the performance of repeated reocclusion and technical failure. Following EVT failure, bypass surgery was performed at the patient's and family's wish, although major amputation and palliative analgesia were feasible alternatives. The clinical outcomes of inframalleolar bypass to the plantar artery branches or LTA have been reported. ${ }^{5,6)}$ There were 2 early graft failures within 30 days $(11 \%)$, and the rates of primary patency, secondary patency, limb salvage, and the patient's survival were $67 \%, 70 \%, 75 \%$, and $91 \%$ at 12 months, respectively. ${ }^{5)}$ Although the patient survival was equivalent to that with dorsal pedal bypasses, the other rates were significantly inferior to those of dorsal pedal bypasses. While the present patient died suddenly from an unknown cause 8 months after the operation, the primary patency of the bypass graft and limb salvage was successfully achieved for the remainder of his life. The long-term results of distal bypass to the distal portion of the LTA remain unclear, but such bypass may be an effective alternative to inframalleolar bypass for limb salvage.

\section{Conclusion}

We herein report a case of CLI in a patient who underwent popliteal to distal LTA bypass. Vascular surgeons should consider the distal LTA also as an effective alternative site for infragenicular revascularization.

\section{Acknowledgments}

The authors thank Dr. Brian T. Quinn for providing critical comments on the manuscript.

\section{Disclosure Statement}

The authors declare no conflict of interest.

\section{Author Contributions}

Writing: KS

Critical review and revision: all authors

Final approval of the article: all authors

Accountability for all aspects of the work: all authors

\section{References}

1) Albers M, Romiti M, Brochado-Neto FC, et al. Metaanalysis of popliteal-to-distal vein bypass grafts for critical ischemia. J Vasc Surg 2006; 43: 498-503.

2) Reifsnyder T, Arhuidese IJ, Hicks CW, et al. Contemporary outcomes for open infrainguinal bypass in the endovascular era. Ann Vasc Surg 2016; 30: 52-8.

3) Diehm N, Shang A, Silvestro A, et al. Association of cardiovascular risk factors with pattern of lower limb atherosclerosis in 2659 patients undergoing angioplasty. Eur J Vasc Endovasc Surg 2006; 31: 59-63.

4) Haimovici H. Open surgical exposures of arteries in the lower extremity. In: Ascher E, Veith FJ, Gloviczki P, eds. Haimovici's Vascular Surgery, 6th ed. Chichester: Blackwell, 2012: 372-88.

5) Hughes K, Domenig CM, Hamdan AD, et al. Bypass to plantar and tarsal arteries: an acceptable approach to limb salvage. J Vasc Surg 2004; 40: 1149-57.

6) Brochado-Neto FC, Cury MV, Bonadiman SS, et al. Vein bypasses to branches of pedal arteries. J Vasc Surg 2012; 55 : 746-52. 